\title{
Deutsche Verzichtsmythen
}

\author{
NORBERT REUTER
}

Deutschland gehört zu den reichsten Ländern der Erde. Das Volkseinkommen ${ }^{\bullet}$ ist seit dem Zweiten Weltkrieg kontinuierlich gestiegen und ist heute real rund siebenmal so hoch wie in den 1950er Jahren. Nie waren die Verteilungsspielräume - trotz des massiven Wachstumseinbruchs im Jahr 2009 - so groß wie heute. Und doch dominiert eine Stimmung, wonach „wir“ seit Langem über unseren finanziellen Möglichkeiten leben würden, ein Rückbau des Sozialstaats alternativlos sei und die ganze Gesellschaft sich zukünftig auf Verzicht einstellen müsse. Ein prominenter Vertreter dieser Auffassung ist der konservative Sozialforscher Meinhard Miegel. In seinem jüngsten Buch „Exit. Wohlstand ohne Wachstum“ zieht er aus einer ansonsten lesenswerten Analyse zunehmender Probleme des wirtschaftlichen Wachstums den (Fehl-)Schluss, dass „Abstriche bei Sozialleistungen “ unumgäng lich seien: Der Staat werde „nicht umhinkommen, den breiten Strom materieller Leistungen spürbar einzudämmen. “2 ${ }^{2}$ Ähnliche Verzichtsbotschaften werden bereits seit Jahren von der arbeitgeberfinanzierten „Initiative Neue Soziale Marktwirtschaft (INSM)“ verbreitet. ${ }^{3}$ Ihr geht es freilich nicht um die Frage, welche politischen Schlussfolgerungen niedrige Wachstumsraten nach sich ziehen. Die INSM propagiert Abstriche bei der Lohnentwicklung und Sozialstaatsabbau im Unterschied zu Miegel als Mittel, um den Trend abnehmender Wachstumsraten aufzuhalten und umzukehren. ${ }^{\circledR}$ Gleichwohl bauen beide Argumentationslinien im Wesentlichen auf zwei Begründungszusammenhängen auf, die sich mit den Begriffen Demografie und (Staats-) Verschuldung knapp umreißen lassen.

\section{Der demografische Wandel}

Der Hinweis auf die Alterung der Gesellschaft dient vielfach als Beleg, dass in Zukunft massive soziale Einschnitte notwendig seien. Wenn es immer weniger Menschen im erwerbsfähigen Alter, dafür immer mehr Rentnerinnen und Rentner gibt, dann scheint gar nichts anderes übrig zu bleiben, als Sozialleistungen zu kürzen und zu streichen. Im Zusammenhang mit den anhaltenden Finanzierungsschwierigkeiten bei der Pflegeversicherung waren jüngst wieder Karikaturen in der Presse zu finden, die schon bei der Einführung der Rente mit 67 kursierten. Da werden für die Gegenwart viele junge Menschen gezeigt, die symbolisch wenige Alte mühelos tragen. Für die Zukunft wird ein ganz anderes Bild gezeichnet: Dann brechen die verbliebenen Jungen unter einer drastisch gestiegenen Zahl an Alten buchstäblich zusammen.

Hat man dieses Bild erst mal verinnerlicht, erscheinen konkrete soziale Verschlechterungen als alternativlos - von der Rente mit 67 über Kürzungen bei den Sozialleistungen und Personalabbau im öffentlichen Dienst bis hin zur Privatisierung von Gesundheit und Pflege. Wer will sich schon dem Vorwurf aussetzen, Fakten nicht zur Kenntnis zu nehmen? Bereits 2003 hatte der damalige Bundeskanzler Gerhard Schröder dieser Entwicklung verbal den Weg geebnet: „Und wir müssen anerkennen und aussprechen, dass die Altersentwicklung unserer Gesellschaft, wenn wir jetzt nichts ändern, schon zu unseren Lebzeiten dazu führen würde, dass unsere vorbildlichen Systeme der Gesundheitsversorgung und der Alterssicherung nicht mehr bezahlbar wären. Was wir heute beweisen müssen, ist der Mut, Neues zu wagen. Dabei werden wir uns von manchem, was uns lieb - und leider auch: teuer - geworden ist, verabschieden müssen. “ $\ominus$

\section{Die Bedeutung der Produktivitäts- entwicklung}

Aber ist es wirklich so, dass es in Zukunft weniger zu verteilen gibt, vieles aufgrund der Schrumpfung und Alterung unserer Gesellschaft „nicht mehr bezahlbar" ist, wir uns insgesamt weniger leisten können? Ob etwas „bezahlbar“ ist und bleibt, hängt tatsächlich nicht nur von der Zahl der Erwerbstätigen, sondern ganz wesentlich von der Produktivität der Arbeit ab. Aufgrund technologischer und organisatorischer Entwicklungen steigert sich die Wertschöpfung je Arbeitsstunde kontinuierlich. Betrug 1991 das Bruttoinlandsprodukt (BIP) je Erwerbstätigen stunde noch rund $29 €$, so waren es 2011 - trotz zwischenzeitlicher Krise - bereits knapp $40 €$ (jeweils zu Preisen des Jahres 2000). Berücksichtigt man diese Entwicklung - ergänzt also die demografische durch eine ökonomische Analyse -, kommt man zu ganz anderen Ergebnissen als es die Verzichtsbotschafter verkünden. Gleichzeitig entschärft sich - wie im Folgenden deutlich werden wird - der Konflikt zwischen Ökologie und Ökonomie.

Legt man eine mittlere Variante der aktuellen, bis 2060 reichenden Bevölkerungsvorausberech nung des Statistischen Bundesamtes zugrunde ${ }^{\circledR}$ und unterstellt, dass bei konstanter Erwerbstätigenquote (d.h. auch in Zukunft sind wie heute nur 75 \% der 15- bis 65-Jährigen erwerbstä-
- Das Volkseinkommen ist die Summe aus allen Arbeitnehmerentgelten und allen Unternehmens- und Vermögenseinkommen.

(2) Miegel, M. (2010): Exit: Wohlstand ohne Wachstum, Berlin, S. $202 f$.

(3) Vgl. Reuter, N. (2001): Arbeitgeber-Marktwirtschaft", in: Blätter für deutsche und internationale Politik 46 (10), S. 1167-1170.
(4) Den Zusammenhang von Wachstum und Wohlstand soll auch die Anfang 2011 eingesetzte Enquete-Kommission „Wachstum, Wohlstand, Lebensqualität" des Deutschen Bundestages untersuchen. Siehe http://www.bundestag.de/bundestag/ausschuesse17/gremien/enquete/ wachstum/index.jsp.

(5) Rede auf dem SPD-Sonderparteitag, 1. Juni 2003 in Berlin.
6 Unterstellt ist hierbei, dass die Geburtenhäufigkeit bei 1,4 Kindern je Frau bleibt, die Lebenserwartung bei Geburt bis 2060 um acht Jahre bei Männern und um sieben Jahre bei Frauen steigt und das jährliche Wanderungssaldo sich auf plus 100.000 Personen beläuft. Dann leben im Jahr 2060 statt heute rund 81 Mio. nur noch knapp 65 Mio. Menschen in Deutschland, vgl. Statistisches Bundesamt (2009): Bevölkerung Deutschlands bis 2060. 12. koordinierte Bevölkerungsvorausberechnung, Wiesbaden. 
tig) die Produktivität je Erwerbstätigem um 1 \% pro Jahr zunimmt, wird - selbst unter diesen sehr restriktiven Annahmen - das Verteilungsvolumen über die nächsten Dekaden nicht kleiner. Zwar würde ab 2020 das BIP nahezu stagnieren (das gesamtwirtschaftliche jährliche Wachstum läge bis $2060 \mathrm{im}$ Durchschnitt nur noch zwischen 0,1 und $0,2 \%$ pro Jahr), dies wäre jedoch ein aus ökologischen Gründen zu begrüßender Effekt. Denn dann würde bei gleichzeitig steigender Ressourcenproduktivität und verbesserten Umwelttechniken auch die Umweltbelastung absolut zurückgehen. Pro Kopf steigt das BIP dennoch kontinuierlich an: von heute rund $31.000 €$ über gut $33.000 € \mathrm{im}$ Jahr 2030 auf fast $42.000 € \mathrm{im} \mathrm{Jahr}$ 2060, was einen realen Pro-Kopf-Anstieg um gut $35 \%$ bedeuten würde. ${ }^{\circledR}$ Mit anderen Worten: Die abnehmende Zahl an Erwerbstätigen würde durch deren zunehmende Produktivität bei gleichzeitig sinkender Gesamtbevölkerung mehr als wettgemacht. ${ }^{8}$

Tatsächlich dürften die genannten Werte eher die Untergrenze markieren. Wenn Arbeitskräfte aufgrund der demografischen Entwicklung knapp werden, wird aller Voraussicht nach auch die Erwerbstätigenquote zunehmen, zumal heute immer noch weit über 4 Mio. Menschen einen Arbeitsplatz suchen und viele Teilzeitbeschäftige länger arbeiten wollen. Bereits bei einem Anstieg der Erwerbstätigenquote auf $80 \%$ würde sich das BIP pro Kopf bis 2030 auf $35.500 €$ erhöhen, bis 2060 sogar auf gut $44.000 €$, was einem realen Anstieg um knapp $45 \%$ gegenüber heute entsprechen würde. In einer optimistischeren Variante ${ }^{\ominus}$ - die Produktivität steigt trotz alternder Erwerbstätiger wie im Durchschnitt der letzten 20 Jahre auch zukünftig um 1,8 \% pro Jahr und das Erwerbstätigenpotenzial der 15- bis 65-Jährigen wird zu $80 \%$ ausgeschöpft -, steigt das BIP pro Kopf der Bevölkerung bis 2030 kontinuierlich auf $41.500 €$ und bis 2060 sogar auf knapp $66.000 €$, was mehr als eine Verdoppelung gegenüber heute bedeuten würde.

Aussagen über derart lange Zeiträume sind zweifellos mit großen Unsicherheiten behaf- tet. Dennoch lässt sich mit diesen Projektionen zeigen, dass die These, in Zukunft sei wegen der Alterung unserer Gesellschaft vieles nicht mehr bezahlbar, implizit unterstellt, dass zukünftig kein oder nur noch ein minimaler Produktivitätsfortschritt zu verzeichnen sein wird. Hierfür allerdings gibt es keine vernünftige Begründung, womit auch die Annahme sich zukünftig verengender Verteilungsspielräume ins Wanken gerät. ${ }^{\oplus}$ Es spricht umgekehrt vieles dafür, dass die Verteilungsspielräume trotz des demografischen Wandels größer werden, denn eine höhere Produktivitätssteigerung als die unterstellten 1,0 \% pro Jahr und ein Anstieg der Erwerbstätigenquote würden zusätzliche Spielräume etwa auch für Arbeitszeitverkürzung eröffnen („,kürzere Vollzeit für alle“). Aus ökologischer Sicht wäre dies zweifellos der Königsweg: Die höhere Produktivität würde für größeren Wohlstand durch mehr Freizeit genutzt.

\section{Aufgaben der Verteilungspolitik}

Sollte die tatsächliche Entwicklung tendenziell so wie in der Projektion unterstellt verlaufen, sind damit jedoch keineswegs alle Probleme gelöst. Im Gegenteil! Zwar ist hiermit der vermeintlich alternative Sachzwang, den „Gürtel enger schnallen zu müssen“ obsolet. Doch das Verteilungsproblem stellt sich in neuer Dimension. Politisch muss dafür gesorgt werden, dass der steigende gesellschaftliche Reichtum auch bei allen ankommt. Dass 2030 statistisch $33.000 €$ oder gar über $41.000 €$ - je nach Annahmen für jede und jeden real zur Verfügung stehen, $206042.000 €$ oder sogar $66.000 €$, sagt nichts über die tatsächliche Verteilung aus. Wenn die seit Jahren auffällige Tendenz zur Einkommensund Vermögenskonzentration anhält, verfestigt sich das heute schon zutage tretende Problem zunehmender sozialer Ungleichheit: Während eine kleine Elite sich immer mehr des wachsenden gesellschaftlichen Reichtums aneignet, wird die Mehrheit von der Wohlstandsentwicklung - beim Einkommen wie bei der Arbeitszeit - abgekoppelt.
Die drohenden Verteilungskonflikte verweisen auf die wachsende Bedeutung von Politik, vor allem von Verteilungspolitik. Durch sie muss sichergestellt werden, dass alle am wachsenden gesellschaftlichen Reichtum partizipieren können. Dies ist zweifellos eine in ihrer Dimension nicht zu unterschätzende Aufgabe für die politisch Verantwortlichen. Aber es ist ein grundlegend anders gelagertes Problem, ob etwas nicht da ist (und deswegen Sachzwänge schafft), oder ob es lediglich ein - wenn auch massives - Verteilungsproblem gibt. Letzteres ist - soweit gewollt - politisch gestalt- und lösbar.

\section{Die Staatsverschuldung im gesamtwirtschaftlichen Kontext}

Die zweite zentrale und vor allem im Zusammenhang mit der Euro-Krise gebetsmühlenartig vorgebrachte Begründung, warum es keine Alternative zum universellen Verzicht gibt, ist die Staatsverschuldung. Sie gilt als ultimativer Beweis, dass auch der deutsche Staat über seine Verhältnisse gelebt hat. Immer wieder wird hier von interessierter Seite auf den privaten Haushalt verwiesen. Wenn dort mehr ausgegeben als eingenommen werde, bleibe zum Sparen und zum Verzicht auch keine Alternative.

Der Staat ist mit einem privaten Haushalt jedoch nicht vergleichbar. Im Unterschied zum privaten Haushalt kann der Staat nicht nur seine Ausgaben, sondern - über die Steuer- und Abgabenpolitik - auch seine Einnahmen regulieren. Zudem haben staatliche Ausgaben im Unterschied zu privaten unmittelbar Auswirkungen auf die Einnahmen, da ein Teil der Ausgaben sofort in Form von Steuern und Abgaben wieder dem Staat zufließt. Die Verschuldung des Staates hat zudem eine ganz wesentliche Funktion im Rahmen des Geld- und Finanzierungskreislaufs einer Volkswirtschaft. ${ }^{\text {(D) }}$ Bei der Debatte um die Staatsverschuldung wird systematisch ausgeblendet, dass Staatsschulden immer Vermögen in gleicher Höhe gegenüberstehen. Erst vor diesem Hintergrund erschließt sich die ,gesamtwirtschaftlich produktive Rolle
(7) Bei diesen und den folgenden Ergebnissen handelt es sich um reale Werte, inflationäre Entwicklungen würden sie entsprechend erhöhen.

8 Eine Abbildung, die diese Berechnungen veranschaulicht, kann beim Autor angefordert werden.

(9 Diese ökonomisch optimistischere Variante wäre zweifellos eine ökologisch pessimistischere, denn der höhere Anstieg der Pro-Kopf-Einkommen wäre mit einem weiteren, wenn auch moderaten BIP-Wachstum von langfristig durchschnittlich knapp einem Prozent pro Jahr verbunden.

(1) Unter den gewählten Annahmen ergibt sich eine kritische Grenze erst dann, wenn der Produktivitätsfortschritt auf 0,5\% pro Jahr einbrechen und die Arbeitslosigkeit genauso hoch bleiben würde.
Dann würde das BIP pro Kopf in der Dekade nach 2020 aufgrund der demografischen Entwicklung leicht zurückgehen, bevor es ab 2040 wieder steigen würde.

(11) Vgl. zu der Argumentation auch Flassbeck, H./ Spiecker, F. (2011): Der Staat als Schuldner - Quadratur des Bösen?, in: Wirtschaftsdienst 91 (7), S. $472-480$. 
der Staatverschuldung “(1): Sie trägt dazu bei, den traditionell hohen Finanzierungsüberschuss der privaten Haushalte - also deren Ersparnisse wieder zurück in den nationalen Wirtschaftskreislauf zu führen. Seit 2000 betrug die Ersparnis aller privaten Haushalte in Deutschland Jahr für Jahr im Durchschnitt knapp $130 \mathrm{Mrd}$. €. Allein für den Zeitraum von 2000 bis 2011 ergibt dies kumuliert eine Summe von über 1,5 Bill. $€$.

Überschüssige Einkommen lassen sich aber nur anlegen bzw. im ökonomischen Sinn „sparen“, wenn im gleichen Umfang andere bereit sind, sich zu verschulden. Deshalb stehen jedem Finanzierungsüberschuss bzw. jeder Ersparnis notwendigerweise Schulden im gleichen Ausmaß gegenüber. Kennzeichnend für die Unternehmen war in der Vergangenheit, dass sie in ihrer Gesamtheit große Teile der privaten Ersparnis über Kreditaufnahme und daraus finanzierte Investitionen in den nationalen Wirtschaftskreislauf zurückgeführt haben. Dies hat sich 2004 grundlegend geändert. Seitdem weist der Unternehmenssektor vor allem wegen stark gestiegener Gewinne Jahr für Jahr hohe Finanzierungsüberschüsse auf. Vom Nachfrager nach Kapitel sind die Unternehmen - wie die privaten Haushalte - zum Anbieter von Kapital geworden. Gleichzeitig ist der deutsche Staat im Zuge umfassender Konsolidierungs- und Kürzungsmaßnahmen zunehmend als Nachfrager nach Krediten ausgefallen. Die Folge: Für immer mehr Kapital fand sich im Inland kein Schuldner mehr, sodass zunehmend Kapital ins Aus-

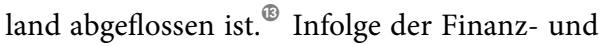
Wirtschaftskrise hat sich dies nach 2008 erst einmal wieder geändert, da sich der Staat zur Bekämpfung der Wirtschaftskrise erneut stärker verschuldet hat. ${ }^{(4)}$ Sollte der Bund die Vorgaben der Schuldenbremse einhalten und es ihm gelingen, seine Neuverschuldung ab 2016 auf maximal 0,35 \% des BIP oder knapp 10 Mrd. $€$ pro Jahr herunterzufahren, die Bundesländer wie gefordert sogar auf null, wird der Staat zukünftig als gesamtwirtschaftlich relevanter Schuldner allerdings praktisch völlig ausfallen.

Ein Ausgleich zwischen Gläubigern und Schuldnern wird künftig dann entweder den Rückgang der Ersparnisse der privaten Haushalte, die Rückkehr des Unternehmenssektors zur umfassenden Kreditaufnahme und/oder einen weiter steigenden Kapitalabfluss ins Ausland erfordern. Das Bedürfnis der privaten Haushalte nach Ersparnisbildung - nicht zuletzt aufgrund des Drucks zur privaten Altersvorsorge - wird jedoch hoch bleiben. Einen Rückgang der Ersparnisbildung der privaten Haushalte dürfte daher vor allem durch eine andere Steuer- und Vermögenspolitik zu erreichen sein, die insbesondere reiche Haushalte mit hohen Sparquoten trifft. Bleiben die Ersparnisse der privaten Haushalte auf dem gegenwärtigen Niveau und sollten die Unternehmen nicht wieder anfangen, sich deutlich zu verschulden - wofür angesichts der schwachen Binnennachfrage und der nachlassenden Exportdynamik wenig spricht bliebe in der Tat nur das Ausland. Mit der dann weiter zunehmenden Kreditgewährung an das Ausland wäre aber ein weiterer Entzug von Binnennachfrage verbunden - mit wiederum negativen Auswirkungen auf die wirtschaftliche Entwicklung.

\section{Das Einnahmeproblem des Staates}

Die Haushalts- und Verschuldungsproblematik mit einer Erhöhung der staatlichen Einnahmen anzugehen, ist auch deshalb notwendig, weil Deutschland seit Langem ein Einnahme- und gerade kein Ausgabenproblem hat. Dies zeigt sich nicht zuletzt daran, dass Deutschland inzwischen einen extrem „schlanken Staat" hat. Deutschland liegt 2011 laut der Statistiken der Europäischen Kommission mit einer Staatsquote von 45,7 \% deutlich unter dem Durchschnitt aller Euroländer mit 49,4\%. Bei diesem Wert sind allerdings die öffentlichen Sozialversicherungssysteme einbezogen. In anderen Ländern ist die soziale Sicherheit jedoch vielfach privatwirtschaftlich organisiert und spielt insofern bei der Berech nung der Staatsquote keine Rolle. Ohne Berücksichtigung der Sozialversicherungen liegt die sogenannte engere Staatsquote in Deutschland bei lediglich rund $26 \%$. Damit entpuppt sich der vielzitierte "Moloch Staat“ inzwischen als ziemlich schwindsüchtiges Wesen.

Immer neue Steuerreformen, von denen vor allem Reiche und gutverdienende Unternehmen profitierten und profitieren, haben die Einnahmebasis des Staates geschmälert - und den privaten Reichtum weiter erhöht. Allein die Steuerreformen seit 1998 haben in den letzten zehn Jahren zu rechnerischen Steuerausfällen in Höhe von kumuliert über 300 Mrd. € geführt. Begründet wurden die Steuerreformen mit der Notwendigkeit, die Wirtschaft zu entlasten, was zu mehr Wachstum und in der Folge zu höheren Steuereinnahmen führen würde. Tatsächlich ist das Wachstum weiter zurückgegangen, und höhere Schulden wurden notwendig, um die Steuersenkungen zu kompensieren und notwendige Ausgaben finanzieren zu können. ${ }^{(5)}$

Vor dem Hintergrund wäre es angemessen gewesen, eine Steuersenkungsbremse gesetzlich zu verankern, um immer neue Steuergeschenke für eine bestimmte Klientel zu unterbinden und dem Staat die notwendigen Einnahmen zu sichern. Stattdessen wurde die Schuldenbremse beschlossen und zum 1. August 2009 im Grundgesetz verankert.

Die Schuldenbremse bedeutet freilich keineswegs, dass eine Vermeidung von neuen Schulden über Ausgabensenkungen zu erfolgen hat. Über Alternativen - etwa eine Erhöhung der Steuereinnahmen - wurde jedoch nicht einmal ansatzweise in der Regierung diskutiert. Deshalb wirkt die Schuldenbremse de facto wie eine Ausgabenbremse. Die schwarz-gelbe Bundesregierung hat dementsprechend mit einem Sparpaket in Höhe von rund $80 \mathrm{Mrd}$. $€$ für den Zeitraum 2011 bis 2014 reagiert, das vor allem zulasten von Arbeitslosen, öffentlich Beschäftigten und sozial Schwachen geht. Vor diesem Hintergrund ist es ein steuerpolitischer Skandal, dass bereits weitere Steuersenkungen von der Regierungskoalition beschlossen worden sind, von denen erneut vor allem Besserverdienende profitieren.

Damit scheint eine Strategie aufzugehen, die Peter Bofinger, Mitglied des Sachverständigenrats zur Begutachtung der gesamtwirtschaftlichen Entwicklung, bereits 2008 beschrieben hat: „Wenn man die Rolle des Staates beschneiden möchte, muss man ihm seine finanziellen Ressourcen entziehen. [...] In einem ersten Schritt werden umfangreiche Steuerentlastungen vorgenommen. [...] Bei unveränderten Ausgaben ergibt sich dadurch eine steigende Neuverschuldung. Wenn man gleichzeitig in der Bevölkerung eine hohe Angst vor der Staatsverschuldung schürt, wird alsbald ein hoher politischer

(12) Reuter, N. (2003): Generationengerechtigkeit als Richtschnur der Wirtschaftspolitik?, in: Butterwegge, Ch./Klundt, M. (Hrsg.): Kinderarmut und Generationengerechtigkeit. Familien- und Sozialpolitik im demografischen Wandel, Opladen, S. 87.

(13 Bisheriger Höhepunkt war eine "Nettokreditgewährung an die übrige Welt", also Kapitalabfluss, in Höhe von 186 Mrd. € im Jahr 2007, vgl. Deutsche Bundesbank (2011): Ergebnisse der gesamtwirtschaftlichen Finanzierungsrechnung für Deutschland 2005 bis 2010, Frankfurt a.M., Tab. 1, S. 23

(44) Eine Abbildung, die diese Entwicklung veranschaulicht, kann beim Autor angefordert werden.

(5) Vgl. hierzu ausführlich Leibiger, J. (2011): Staatsverschuldung in der Ära des Neoliberalismus, in: Berliner Debatte Initial 23 (2), S. 30-47. 
Druck für die Ausgabenkürzungen geschaffen. Deren Umsetzung stellt dann die zweite Stufe des Ressourcenentzugs dar [...].“(®)

\section{Teilhabe statt Verzicht}

Die Haushalts- und Finanzierungsprobleme lassen sich nur mit einer Wende in der Steuerpolitik angemessen und sachgerecht lösen. Vor allem Reiche, Vermögende und gutverdienende Unternehmen müssen wieder stärker zur Finanzierung staatlicher Ausgaben herangezogen werden. Hiermit würde nicht nur die gesamtwirtschaftliche Ersparnis (und damit die Notwendigkeit von Schulden) reduziert, vielmehr würden zusätzliche Einnahmen zur Finanzierung gesamtgesellschaftlich notwendiger Aufgaben generiert. Erwähnt seien hier vor allem überfällige zusätzliche Ausgaben für Bildung, Infrastruktur und den sozial-ökologischen Umbau. ${ }^{\text {(i) }}$

Die Vorschläge für eine solche Steuerpolitik liegen seit Langem auf dem Tisch. Ver.di hat zum Beispiel mit dem „Konzept Steuergerechtigkeit“ vorgerechnet, dass unter anderem mit einer stärkeren Besteuerung von hohen Einkommen, Gewinnen, Vermögen und Finanztransaktionen jährliche Mehreinnahmen in Höhe von 75 Mrd. € erzielt werden könnten. ${ }^{\circledR}$ Dies wären notwendige Bedingungen, damit das wachsende Volkseinkommen zur Finanzierung gesamtgesellschaftlicher Aufgaben genutzt werden kann.
Zur Korrektur der zunehmend schiefer gewordenen Primärverteilung, also der Aufteilung des Volkseinkommens direkt bei der Entstehung auf einerseits Arbeits- und andererseits Gewinnund Vermögenseinkommen, müssen zudem die Rahmenbedingungen auf dem Arbeitsmarkt verbessert werden. Dazu gehört vor allem eine Reform der Arbeitsmarktgesetze, damit Arbeitslose nicht gezwungen sind, unterwertige, schlecht bezahlte Jobs annehmen zu müssen. Dazu gehören auch die geschlechterübergreifende Gewährleistung von gleichem Lohn für gleiche Arbeit („equal pay“) und die Einführung des gesetzli-

(16) Bofinger, P. (2008): Das Jahrzehnt der Entstaatlichung, in: WSI Mitteilungen 61 (7), S. 351, http:// www.boeckler.de/wsimit_2008_07_bofinger.pdf.

(1) Vgl. hierzu Arbeitsgruppe Alternative Wirtschaftspolitik (2012): Memorandum 2012. Europa am Scheideweg - Solidarische Integration oder deutsches Spardiktat, insbes. S. 260ff., Köln.

(8) Vgl. ver.di/Bereich Wirtschaftspolitik (2009): Konzept Steuergerechtigkeit. Gerechte Steuern für mehr Zukunftsvorsorge, Berlin; vgl. auch GEW/ Hauptvorstand (2011): Richtig gerechnet: Gute Bildung ist finanzierbar. Das steuerpolitische Konzept der Gewerkschaft Erziehung und Wissenschaft, Frankfurt a.M.

(19) Vgl. Sterkel, G./Wiedemuth, J. (2011): Neue solidarische Tarifpolitik. Dimensionen eines Richtungswechsels in der Verteilungsfrage, in: Sozialismus (9), S. $31 \mathrm{f}$. chen Mindestlohns. Auf dieser Grundlage ließen sich dann auch wieder (mindestens) verteilungsneutrale Lohnsteigerungen durchsetzen. ${ }^{(1)}$

Die zunehmende Ungleichverteilung von Einkommen und Vermögen in Deutschland unterstreicht die Dringlichkeit dieser Maßnahmen. Deren Umsetzung würde dazu beitragen, dass die breite Bevölkerung am auch zukünftig weiter steigenden gesellschaftlichen Reichtum teilhaben könnte - statt mit immer neuen Verzichtsmythen auf ein „Gürtel-enger-schnallen“ und die damit verbundene weitere Umverteilung von unten nach oben eingeschworen zu werden.

\section{AUTOR}

NORBERT REUTER, PD Dr., ist Ökonom im Bereich Wirtschaftspolitik beim Bundesvorstand der Vereinten Dienstleistungsgewerkschaft (ver.di), lehrt Volkswirtschaftslehre an der Rheinisch-Westfälischen Technischen Hochschule (RWTH) Aachen und ist sachverständiges Mitglied der Enquete-Kommission „Wachstum, Wohlstand, Lebensqualität" des Deutschen Bundestages. Arbeitsschwerpunkte: Strukturwandel, Verteilung, demografische Entwicklung.

norbert.reuter@verdi.de 